\title{
Quantitative Characterization of Features Affecting Crack Path in a Directionally Solidified Superalloy
}

\author{
Matthew D. Trexler, and Thomas H. Sanders Jr. \\ School of Materials Science and Engineering, Georgia Institute of Technology, \\ Atlanta GA 30332, United States of America
}

Keywords: nickel alloys, fractography, laser confocal microscopy, tensile properties

\begin{abstract}
Quantitative metallographic and fractographic methods were used to determine that the fracture path during tensile tests of GTD-111 DS (directionally solidified) superalloy is influenced by the presence of $(\mathrm{Ti}, \mathrm{Ta}) \mathrm{C}$ carbides. Combined profilemetric and confocal microscopic techniques were used to determine the true surface areas of carbides on the fracture surfaces of both longitudinally and transversely oriented tensile specimens. The results show that the local area fraction of carbides on the longitudinally oriented fracture surfaces of specimen tested over a range of temperatures is up to five times greater than the overall bulk area volume fraction of carbides in the material. The transverse case also shows higher local area fractions than the bulk material, although to a lesser extent. The results show that the longitudinal samples exhibit a stronger preference to failing at carbides when compared to the transverse orientation. Additionally, the carbides were observed to fail by cracking rather than debonding from the matrix phase.
\end{abstract}

\section{Introduction}

GTD-111 DS is a directionally solidified Ni superalloy that is used in high temperature turbine engine components. The columnar grain structure of the alloy leads to anisotropic tensile properties, depending on the direction of loading with respect to the oriented grain structure. Tensile tests were conducted by Ibanez [1] at several temperatures ranging from room temperature to $881^{\circ} \mathrm{C}\left(1600^{\circ} \mathrm{F}\right)$ where longitudinal (LT) oriented specimen are samples in which the loading axis was parallel to the columnar grains, and transversely (TL) oriented specimens are loaded perpendicular to the columnar grains. Figure 1 shows the plotted results of yield stress versus temperature. The yield strength shows a maximum around $860^{\circ} \mathrm{C}\left(1400^{\circ} \mathrm{F}\right)$. This has also been observed in other DS superalloys [2].

It has been suggested that the increase in strength with increased temperature, referred to as the anomalous behavior, with increasing temperature is attributed to the high ( $>50 \%$ volume fraction) of the precipitate phase, $\gamma^{\prime},[3-5]$ as $\gamma^{\prime}$ experiences an increase in strength as temperature is increased. The strength gain is attributed to a change in deformation mechanisms from octahedral slip of the precipitate phase at temperatures below $600^{\circ} \mathrm{C}$ to cubic slip at temperatures greater than $600^{\circ} \mathrm{C}$ [3-5].

Calculated values of fracture toughness (tests also conducted by Ibanez [1]) are plotted in Figure 2. According to Ibanez [1] there is little change in toughness observed between room temperature and $649^{\circ} \mathrm{C}\left(1200^{\circ} \mathrm{F}\right)$ where there is an abrupt drop in fracture toughness. The fracture toughness curve reaches a minimum at $860^{\circ} \mathrm{C}\left(1400^{\circ} \mathrm{F}\right)$ and then increases with increasing temperature until $881^{\circ} \mathrm{C}\left(1600^{\circ} \mathrm{F}\right)$. The toughness values found in the transverse orientation are higher than those in the longitudinal orientation for every temperature considered [1].

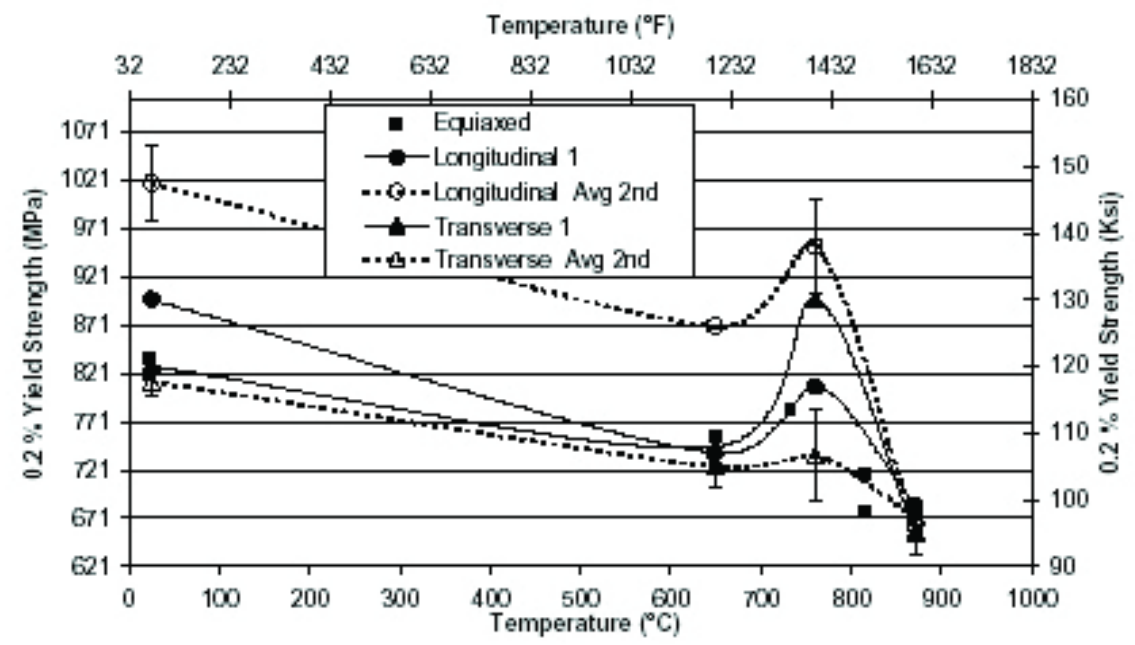

Figure 1: Yield strength as a function of temperature for the different batches of DS GTD-111 in the longitudinal and transverse orientations. [1] 


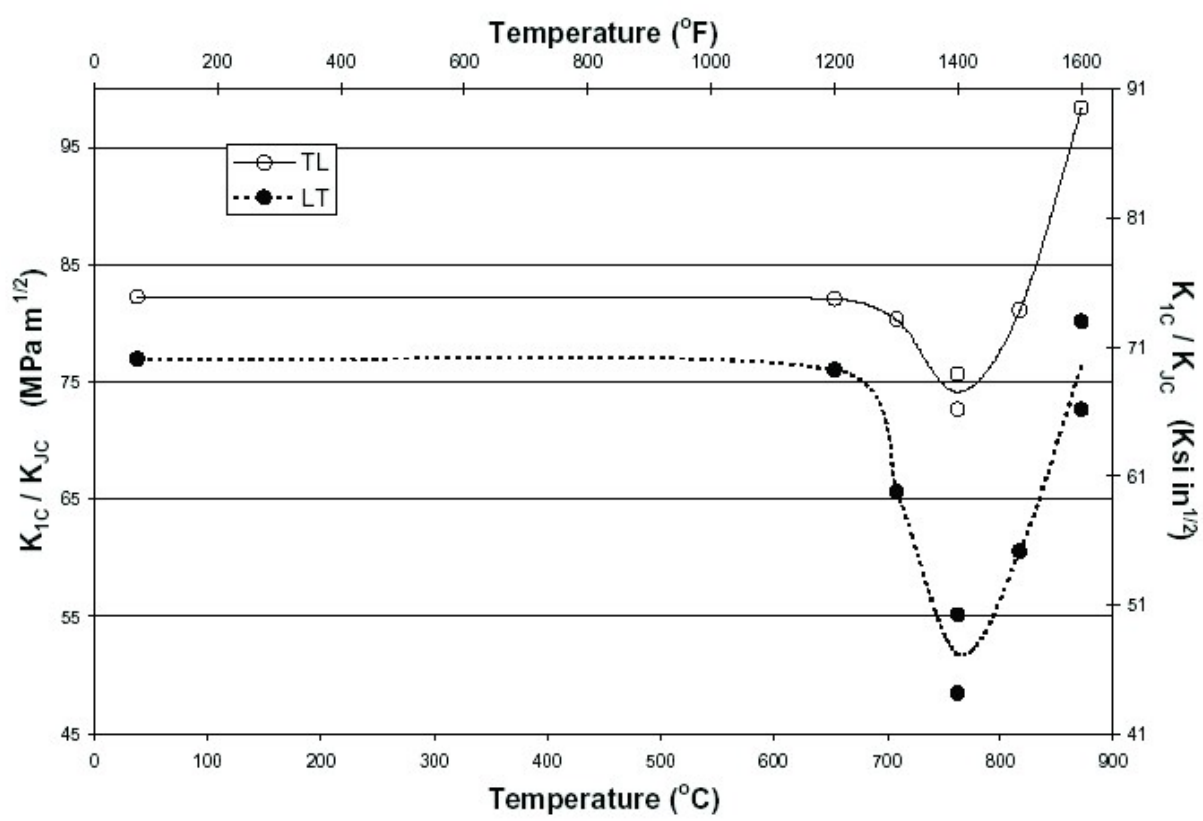

Figure 2: Fracture toughness for DS GTD-111 as a function of temperature in the LT and TL orientations [1].

The drop in fracture toughness is also attributed to a change in deformation mechanisms from octahedral slip of the precipitate phase at temperatures below $600^{\circ} \mathrm{C}$ to cubic slip at temperatures greater than $600^{\circ} \mathrm{C}[3-5]$. This can be further supported by plotting fracture toughness as a function of yield strength (Figure 3 ), which shows that as the yield strength of GTD-111 increases as a function of temperature, the fracture toughness decreases.

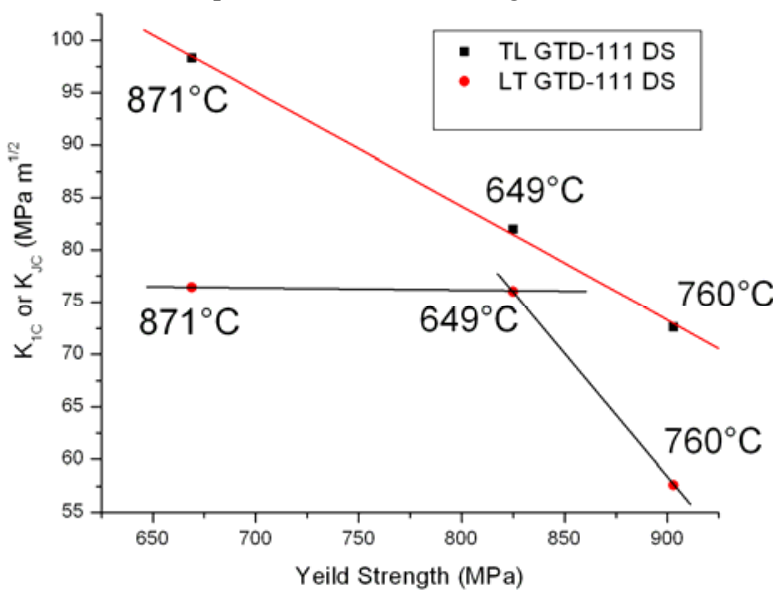

Figure 3: Plot of fracture toughness as a function of yield strength for both TL and LT GTD-111 DS samples tested at $649^{\circ} \mathrm{C}\left(1200^{\circ} \mathrm{F}\right) 860^{\circ} \mathrm{C}\left(1400^{\circ} \mathrm{F}\right)$, and $881^{\circ} \mathrm{C}\left(1600^{\circ} \mathrm{F}\right)$ [1].

Fracture surface analysis indicates that Ta-rich MC carbides play an important role during fracture. The fracture surfaces show large amounts of brittle fracture, associated with interdendritic carbides that are surrounded by small areas of ductile fracture.

Figure 4 shows a comparison of a fracture surface with a random plane of polish just below the fracture surface. Clearly, there is a higher concentration of carbides at the fracture surface than in the plane of polish.

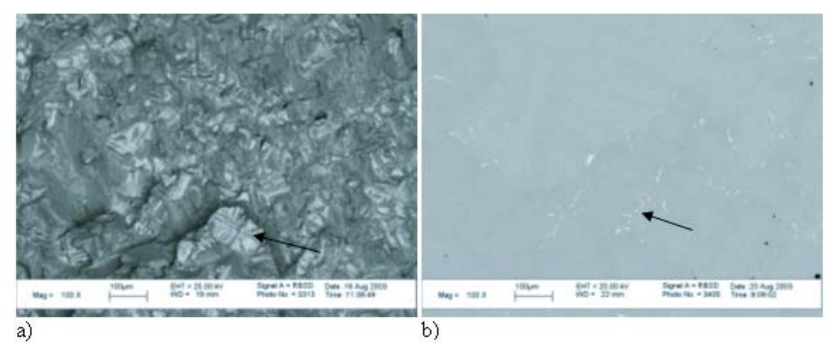

Figure 4: Backscattered electron (BSE) images of a fracture surface (a) and plane beneath fracture (b.) The arrows point to carbides and the test temperature was $860^{\circ} \mathrm{C}\left(1400^{\circ} \mathrm{F}\right)$.

Quantitative stereology has been employed to determine the volume fractions of phases present in bulk GTD-111 DS. Using additional techniques one can compare these bulk features to three-dimensional fracture surfaces. When sectioning mechanical test specimens from partially oriented, large grained materials, such as DS materials, there is often great variability in the number of grains per specimen, particularly between the longitudinal and transverse directions. Figure 5 shows the relative size of a tensile test sample to the slab from which it was machined. It can be seen that if the sample is machined in the direction perpendicular to the growth direction, a larger amount of grains are included in the sample than if the specimen is cut parallel to the growth axis. In fact, it is possible that the longitudinally cut samples may not intersect any grain boundaries at all. 


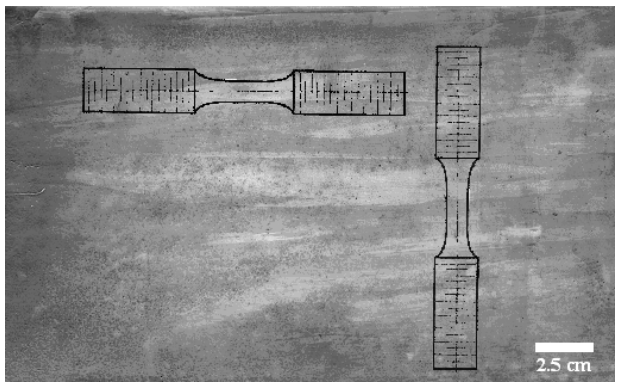

Figure 5: Slab of GTD-111 DS with tensile specimen outline in both the longitudinal and transverse directions. Tensile sample dimensions were $8.62 \mathrm{~cm}$ long with a $2.54 \mathrm{~cm}$ gauge length. The gauge diameter was $0.4 \mathrm{~cm}$ [1].

The quantified number of grain boundaries per unit length can be determined by counting the number of intersections between the grain boundaries and an overlaid set of parallel tests lines on the slab of GTD-111 DS in both the longitudinal and transverse directions. The results of this analysis show transverse tensile specimens have approximately 4 times as many grain boundaries per unit length than longitudinally oriented samples. It has been shown that strength increases as the number of grain boundaries perpendicular to the loading axis decreases [6]. This is important if one assumes grain boundaries that are perpendicular to the loading axis are weaker than grain boundaries parallel to the loading axis and are therefore more likely to cause failure. The relative strengths of the grain boundaries in either direction can be estimated using creep ductility data [6]. Figure 6 shows results obtained by Ibanez [1,3]. The creep ductility of the longitudinally oriented samples is higher than that of the transversely oriented samples, which supports the assumption that transversely oriented grain boundaries are weaker, especially at temperatures above $649^{\circ} \mathrm{C}\left(1200^{\circ} \mathrm{F}\right)$.

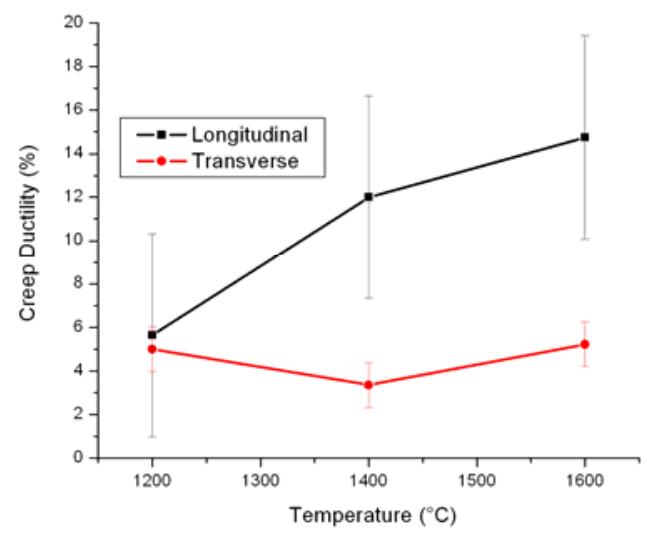

Figure 6: Creep ductility (\%) as a function of temperature DS GTD-111 DS in the longitudinal and transverse directions $[1,5]$.

\section{Experimental Procedure}

The grip sections of each tensile specimen in the transverse (perpendicular to the loading axis) and longitudinal (parallel to the load axis) directions were sectioned after the tests. The sectioned samples were mounted in edge-retaining bakelite mount and were mechanically polished using abrasive SiC paper. Final polishing occurred using alumina suspension in steps from 1.0 to $0.05 \mu \mathrm{m}$. The precipitate phases were identified using a scanning electron microscope (SEM) equipped with an electron dispersive spectroscopy system. To quantitatively characterize the volume fractions of these phases, both optical and SEM imaging were used in conjunction with digital image analysis using standard stereological techniques [8].

To examine the relationship between fracture behavior and precipitate phases, the fracture surface of each tensile sample was examined in the SEM. An image montaging technique was used to provide high magnification images of the entire fracture surface. Once both mating surfaces were imaged using the SEM, the values of the surface roughness parameter, Rs, were determined using profilemetry and confocal microscopy. The directionally solidified nature of the sample oriented parallel to the directionally solidified direction allows accurate quantitative measurements using a single vertical section [8]. To preserve the fracture surface profile during sectioning, an edge retaining mounting material was applied to the sample prior to cutting.

The vertical sections were then polished using the previously described method. Key fractographic parameters such as the linear roughness parameter $\left(R_{L}\right)$, profile structure factor $(\Psi)$, apparent area fraction $\left(\mathrm{A}_{\mathrm{A}}\right)$, surface roughness parameter $(\mathrm{Rs})$, and the true area fraction of phases $(\mathrm{F})$, were determined using well established fractographic methods [8] to correct for the fact that SEM images represent only a projection of 3-D surfaces. This can lead to up to $40 \%$ error when determining area fractions [8].

\section{Quantitative Fractography}

Quantitative fractography was employed to connect fracture behavior to the key microstructural features in the tensile specimens already studied. The goal of the technique was to provide quantitative information on the role of each constituent with respect to fracture path. It has been observed that the relative amount of carbides on the fracture surface appear to be larger than the amount in the bulk material. This suggests that the carbides largely affect crack path. Two methods, profilemetry and an SEM based technique were used to explore this phenomenon quantitatively. A schematic of the two cases is shown in figure 7. A brief discussion follows.
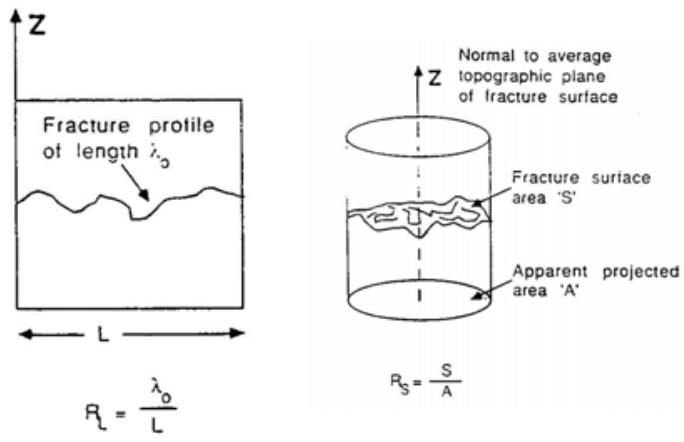

$P_{S}=\frac{S}{A}$

Figure 7: Profile roughness $R_{L}$ and surface $R_{s}[8]$. 


\section{$\underline{\text { Profilometry }}$}

Fracture surfaces, when intersected by a vertical plane or section, yield a line profile of that fracture surface. A trace of the $x$ and $y$ coordinates of this profile can easily be collected using digital image analysis as seen in figure 8 . From these data, several parameters can be determined to characterize the fracture surface. These include: fracture surface roughness, surface anisotropy, and the fraction of the profile through a given microstructural feature [8]. The experimental procedure for obtaining fracture profiles is fairly straight-forward. First, a vertical section perpendicular to the loading axis was chosen. The fracture surface must be plated with metal or polymer to protect the surface during sectioning. The sectioned sample was then mounted, polished, and etched using standard metallographic techniques. Optical and scanning electron microscopy was then used to study the profile.

The profile roughness parameter $\mathrm{R}_{\mathrm{L}}$ is defined as:

$$
R_{L}=\frac{\lambda}{L}
$$

where $\lambda$ is the total length of the fracture profile and $\mathrm{L}$ is the projected length of the profile. The surface roughness parameter was instrumental in allowing the use of SEM based techniques as part of this work [8]. Another attribute that was useful is the fraction of the profile that passes through specific microstructural features, $P_{f}$. The following example illustrates the benefits of such measurements. A two phase material consists of $\beta$ particles in a $\beta$ matrix. The volume fraction of $\beta$ in $\beta$ is $2.0 \% . P_{f}$ for the fracture profile is $25.0 \%$.

Clearly, the fracture path is preferentially following $\beta$ particles. Use of this parameter enables the connection of specific features to the fracture path. Based on qualitative results, it is proposed that cracks preferentially follow the interdendritic regions of the microstructure.

\section{$\underline{\text { SEM based method }}$}

While profilemetry alone is a powerful tool, it can easily be combined with an SEM approach to provide even more information. Most stereological methods can be applied to SEM fractography. However, it is vital to realize that SEM images are merely a projection of a 3-dimensional surface. As a result, the surface area of SEM images cannot be taken as the true surface area of the fracture surface [8]. A correction must be made to account for the lost of 3-D information due to the projection. An analog to the profile roughness parameter, $R_{L}$, is the surface roughness parameter, $R_{S}$, which is the true surface area divided by the apparent projected area.

$$
R_{S}=\frac{S}{A}
$$

To experimentally determine $\mathrm{R}_{\mathrm{S}}$, the data generated by the profilemetry was used. This is done using:

$$
R_{S}=R_{L} \psi
$$

where $R_{L}$ is the profile roughness and is the profile structure factor. The equation depends on the average of the product of the
$\mathrm{R}_{\mathrm{L}}$ and over several vertical sections. The number of vertical sections needed to accurately determine $\mathrm{R}_{\mathrm{S}}$ ranges between one and three [8]. For isotropic fracture surfaces it has been shown that only one section is needed. For structures with a preferred orientation, such as DS materials, the number of sections needed depends on the loading direction of the fracture specimen. A DS material loaded in the longitudinal direction will crack across randomly oriented grains yielding an isotropic fracture surface. When loaded in the transverse direction, however, the fracture surface is anisotropic. More specifically, the dendritic structure of the DS material will yield what is referred to as a ruled surface [8]. In such cases it has been shown that an accurate value of $R_{S}$ can be determined using only three vertical sections oriented 120 degrees from one another. Determining $\mathrm{R}_{\mathrm{L}}$ has already been described. The structure factor can also be calculated from the profile data. $\Psi$ is given by:

$$
\psi=\int\left[\left(\sin \alpha+\frac{\pi}{2}\right)-\alpha \cos \alpha\right] \mathrm{f}(\alpha) \mathrm{d} \alpha,
$$

where $\alpha$ is the angle between a line segment and the vertical axis such that $\alpha$ is between 0 and $\pi$, and $F(\alpha) \operatorname{d} \alpha$ is the distribution function of all line segments having an orientation in the range of $\alpha$ to $(\alpha+d \alpha)$. Since distribution functions are often calculated in a histogram, a method has been developed to solve the integral using a discretized summation [8].

$$
\psi=\sum a_{i} h_{i}
$$

where $a_{i}$ is a coefficient and $h_{i}$ is the fractions of line segments that have an orientation angle, $\alpha$, in the range between 10(i-1) and 10i. The values for $i$ and $a_{i}$ can be found in existing tables. Once $\mathrm{R}_{\mathrm{S}}$ is determined, the projected images acquired in the SEM can be corrected to give the true surface area. SEM analysis can then be used to determine area fraction of the carbides on the fracture surface. The volume fraction of carbides in the bulk material has been determined to be $2 \%$ using BSE imaging and the point counting method. The fracture surfaces of each specimen was imaged using this same BSE technique as BSE imaging yields better compositional contrast [44] prior to sectioning. This was done since sectioning for profilemetry destroys the fracture surface. An areal analysis performed on the SEM fractographs will yield the area fraction of carbides per apparent area. The roughness parameter can then be used to correct this value to the area fraction of carbides per true area $(\mathrm{F})$ :

$$
\mathrm{F}=\frac{\mathrm{R}_{\mathrm{S}}}{\left(\mathrm{R}_{\mathrm{S}}\right)_{\beta}} A_{a}
$$

where $\left(\mathrm{R}_{\mathrm{S}}\right)_{\beta}$ is the surface roughness parameter of the specific inclusion and $\mathrm{A}_{\mathrm{a}}$ is the projected area fraction from the SEM image. Similarly, the number density of carbides on the fracture surface $\mathrm{N}_{\mathrm{f}}$ can be determined using: 


$$
\mathrm{N}_{\mathrm{f}}=\mathrm{N}_{\mathrm{s}} \mathrm{R}_{\mathrm{S}}
$$

where Ns is the number density of carbide found in the SEM micrograph. This must also be corrected by dividing by the roughness parameter Rs. Once again, if the values of $\mathrm{F}$ and $\mathrm{Nf}$ of a given feature are higher than the values in the bulk material, then a preference for crack path can be observed. In the rare case where the area fraction of the feature is less than in the bulk, the feature may retard crack growth [8]. Figure 8 shows an example of a profile and its corresponding digitization which shows how these parameters were determined.
In the case of transverse samples, the fracture surfaces lack the symmetry found in longitudinal specimens. The result is that three vertical sections are needed for profilemetry [8]. Thus, using profilemetry on the transversely oriented samples proved to be impractical given the experimental difficultly of sectioning such small fracture surfaces. To avoid these difficulties and measure the true surface roughness directly, without sectioning, a Leica laser scanning confocal microscope (LSCM) was used to obtain the true surface areas of fracture specimens in both the transverse and longitudinal orientation. The use of LSCM to characterize surface roughness of material is well documented in the literature $[8,8]$. To normalize all of the results, a calibration curve was used to relate the roughness values obtained by LSCM to those determined by profilemetry (Figure 9 ).

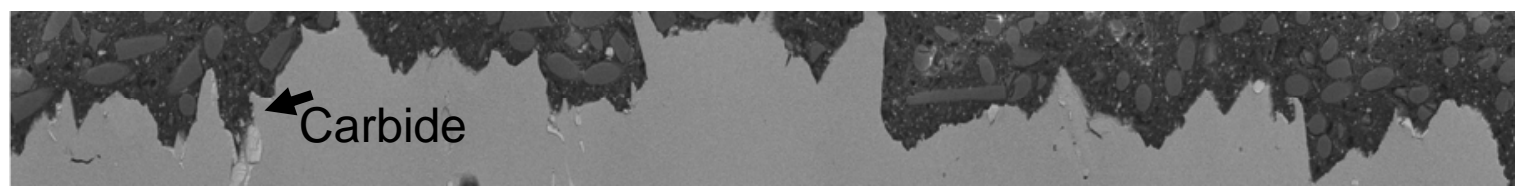

SEM Image $\quad 881^{\circ} \mathrm{C}$

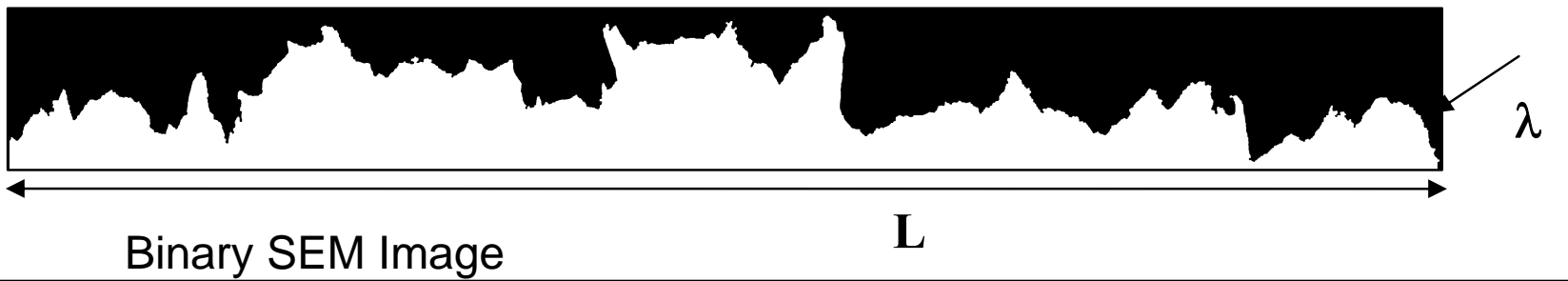

Figure 8: Example of profile and its corresponding binary image. From the image the surface roughness of both the fracture surface $\left(R_{S}\right)$ and the roughness of the carbide $\left(R_{S}\right)_{B}$ can be determined.

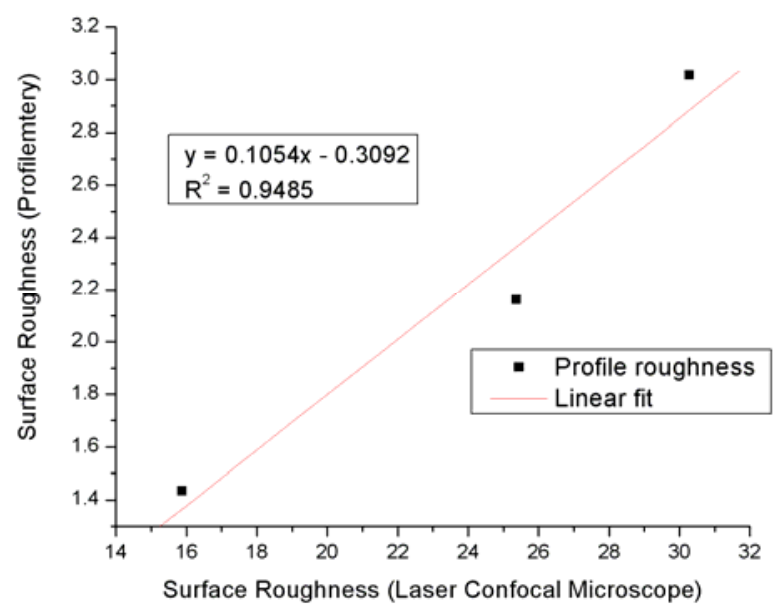

Figure 9: Correction plot for normalized surface roughness of transverse profiles and transverse specimen using laser scanning confocal microscopy. Using the equation $y=0.1054 x-0.3092$ the LSCM specific surface roughness for longitudinal values may be normalized for comparison. 


\section{Results and Discussion}

Stereographic measurements indicated that the major strengthening phase present is $\mathrm{Ni}_{3} \mathrm{Al}$ precipitates range in size from $0.1 \mu \mathrm{m}$ to $1.0 \mu \mathrm{m}$. The volume percent of $\gamma^{\prime}$ in GTD-111 DS was determined to range between $50-60 \%$. This has also been observed in other studies [9-11]. In addition to $\gamma^{\prime},(\mathrm{Ti}, \mathrm{Ta}) \mathrm{C}$ carbides were found, mostly in the interdendritic regions of the material. This can be attributed to the fact that these carbides form early in the casting process and are free to float within the melt [12]. They are then trapped between dendrite arms when the last of the liquid solidifies. The shapes are often referred to as the "Chinese script" morphology due to the fact they resemble Chinese characters [4]. This can be illustrated best in Figure 10 which shows an individual carbide that has been removed from the matrix using a chemical extraction technique in accordance with ASTM standards [13]. The average volume fraction of carbides in bulk GTD-111 DS was determined to be $<0.02(<2 \%)$ and the number density of the carbide was $1.5 \times 10^{-4}$

Oriented Structure Characterization: Number of Grain Boundaries per Unit Length

By placing a set of parallel test lines over the slab shown in Figure 5 , the number of grain boundaries per unit length was determined to be $0.563 \mathrm{~cm}^{-1}$ in the longitudinal direction. In the transverse direction, the number of grain boundaries per unit length was determined to be $2.388 \mathrm{~cm}^{-1}$ suggesting that samples oriented in the transverse direction with respect to the growth axis will have approximately four times as many grain boundaries per unit length than longitudinally oriented samples.

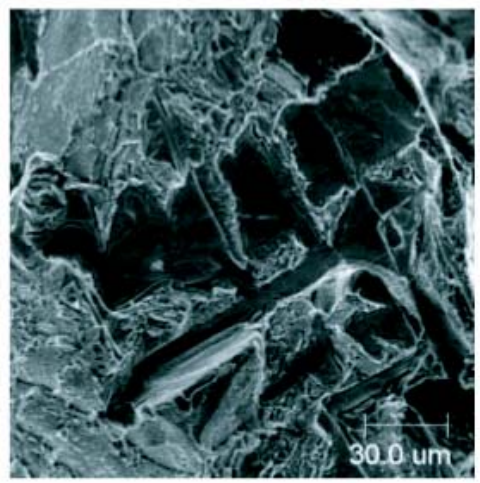

SE Image
Fracture Surface Measurements

Identifying key microstructural features that may influence the fracture behavior in a scanning electron microscope can be difficult due to lack of contrast when using secondary electrons. However, using backscattered electron (BSE) imagining, the compositional contrast between the $\mathrm{Ti}, \mathrm{Ta}$-rich carbides and the Ni matrix makes it easy to identify fracture surface carbides [14]. Figure 11 compares two scanning electron microscope micrographs.

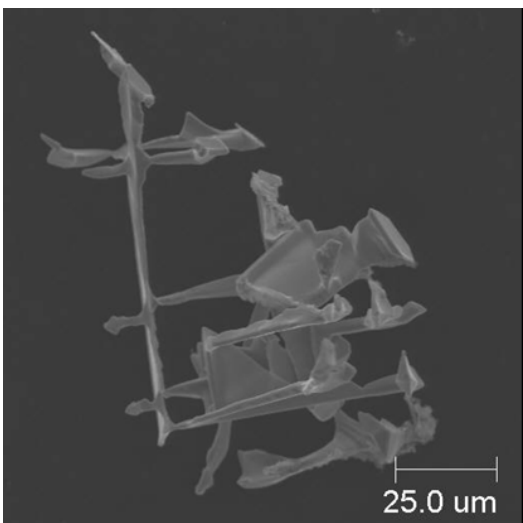

Figure 10: Script carbide chemically extracted from the matrix

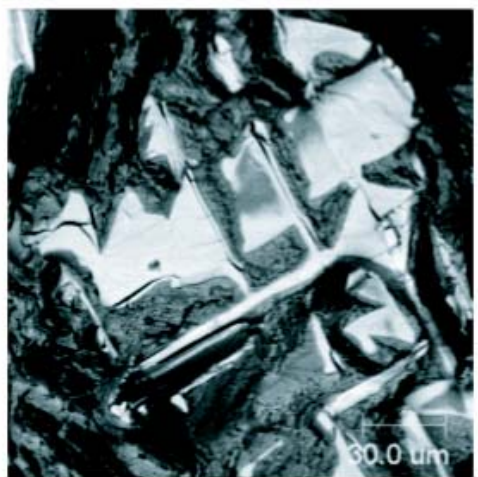

BSE Image

Figure 11: Scanning electron microscope images of fracture surface of tensile specimen tested at $860^{\circ} \mathrm{C}$. The left image is a secondary image (SE) and the right is a BSE image.

In the SE image it is often difficult to distinguish the boundaries between carbides and the matrix. The BSE imaging, however, provides the distinction of clear boundaries of the particle. This is due to the fact that the backscattered coefficient, $\eta$ (the number of backscattered electron/number of incident electrons), increases monotonically with atomic number. This serves as the basis for compositional contrast [16]. Heavier elements, such as Ta will appear brighter than lighter elements, such as the nickel matrix, which will appear dark. The distinct compositional contrast therefore makes it easy to quantify the carbides. This is true in polished samples as well as on the fracture surfaces and was used to quantify these features.

To confirm that these features are Ti, Ta carbide, EDS mapping was employed to mapped the relative amounts of $\mathrm{Ti}, \mathrm{Ta}, \mathrm{Ni}, \mathrm{Cr}$, and C. Figure 11 shows that the areas that are rich in the carbide forming elements $(\mathrm{Ti}, \mathrm{Ta}$, and $\mathrm{C})$ are also deficient the matrix forming elements $(\mathrm{Ni}$ and $\mathrm{Cr}$ ). The results for longitudinal samples (Table I) show that the true area fraction, F, and number density, $\mathrm{N}_{\mathrm{f}}$, on the fracture surfaces at each of the test 
temperatures is significantly higher than the bulk volume fraction and number density respectively.

This suggests that the carbides are greatly affecting the tensile fracture behavior of the longitudinally oriented GTD-111 DS. The average difference between the apparent carbide area fractions $\left(\mathrm{A}_{\mathrm{s}}\right)$ and the true area fractions $(\mathrm{F})$ was approximately $2 \%$ indicating that a fairly low degree of error is introduced when using only the apparent area fractions from the scanning electron microscope images.

The number density also follows a similar trend showing that the greater amount of carbides per unit area is found on the fracture surface of the longitudinally oriented specimen. This can be explained by recalling that grain boundaries are weaker when they are oriented perpendicular to the loading axis, as is the case with transversely oriented samples [14]. In this case the dominant cause of failure is the grain boundary itself, so failure is less likely to be caused by the carbides, resulting in fewer carbides being found on the fracture surface. In the longitudinally oriented case, however, the grains are perpendicular to the loading axis, which maximizes the grain boundary resistance to failure. Now, the grain boundary itself is not the dominant cause of failure; the carbides are responsible for fracture, which explain the greater area fractions of carbides on the fracture surfaces.

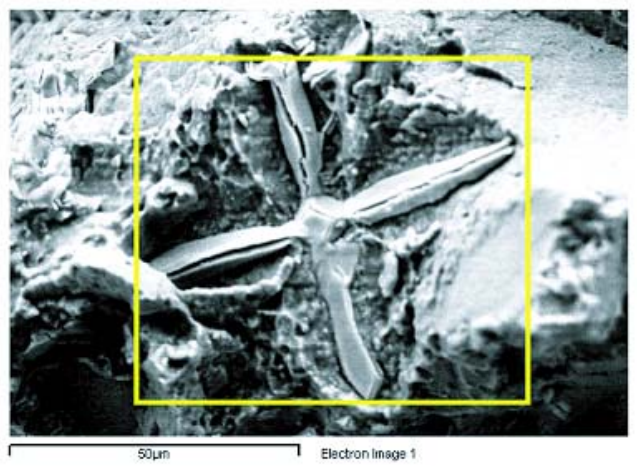

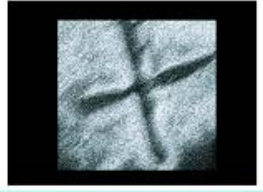

Nickel

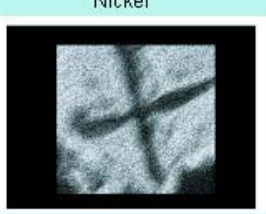

Chromium

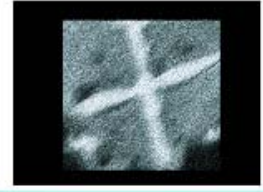

Titanium

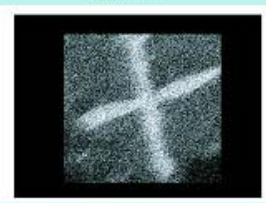

Tantalum

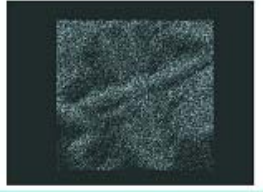

Carbon

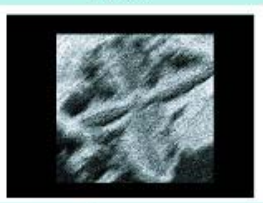

Oxygen

Figure12: X-ray map of fracture surface of tensile specimen tested at $860^{\circ} \mathrm{C}$.

Table I: Summary of the fractographic parameters obtained from longitudinally oriented samples to determine the true area fraction of carbide on the fracture surface, $F$. The values

\begin{tabular}{|c|c|c|c|c|c|c|}
\hline \multicolumn{2}{|c|}{ Temperature } & \multicolumn{5}{|c|}{ Fractography Parameters } \\
\hline${ }^{\circ} \mathrm{C}$ & ${ }^{\circ} \mathrm{F}$ & $\mathrm{R}_{1}$ & $\Psi$ & $\mathrm{R}_{\mathrm{s}}$ & $\mathrm{A}_{\mathrm{s}}$ & $\mathrm{F}$ \\
\hline 21 & 80 & 3.686 & 1.022 & 3.868 & 0.118 & 0.091 \\
\hline 649 & 1200 & 2.814 & 1.258 & 3.413 & 0.133 & 0.132 \\
\hline 860 & 1400 & 2.663 & 1.245 & 3.314 & 0.123 & 0.118 \\
\hline 881 & 1600 & 2.058 & 1.218 & 2.504 & 0.114 & 0.085 \\
\hline
\end{tabular}

Based on Figure 9, a linear relationship was observed and was used to convert the roughness value into similar terms for comparison. Table II summarizes all of the results obtained in this study. Both orientations show higher area fractions of carbides on the fracture surfaces than what can be found in the bulk material. However, on average, the longitudinal orientation shows approximately four times as much carbide area as does the transversely oriented case. In summary, the relative likelihood that either orientation or the presence of carbides would be the

Table II: Summary of the area fraction of carbides and number per unit area of carbides $\mu^{-2}$ for both longitudinal and transverse orientations. The values for $F$ and $N_{f}$ at all temperatures is significantly higher in bulk GTD-111 DS.

\begin{tabular}{|c|c|c|c|c|c|}
\hline \multicolumn{2}{|c|}{ Temperature } & \multicolumn{2}{c|}{ Area fraction of carbides $(\mathrm{F})$} & \multicolumn{2}{c|}{ Number per unit area $\left(\mathrm{N}_{\mathrm{f}}\right)\left(\mu \mathrm{m}^{-2}\right)$} \\
\hline${ }^{\circ} \mathrm{C}$ & ${ }^{\circ} \mathrm{F}$ & Longitudinal & Transverse & Longitudinal & Transverse \\
\hline 21 & 80 & 0.118 & 0.038 & $6.46 \mathrm{E}-04$ & $1.33 \mathrm{E}-04$ \\
\hline 649 & 1200 & 0.133 & 0.016 & $4.65 \mathrm{E}-04$ & $2.93 \mathrm{E}-05$ \\
\hline 860 & 1400 & 0.123 & 0.063 & $4.64 \mathrm{E}-04$ & $6.04 \mathrm{E}-05$ \\
\hline 881 & 1600 & 0.114 & 0.031 & $8.83 \mathrm{E}-04$ & $8.99 \mathrm{E}-05$ \\
\hline
\end{tabular}

dominant cause of failure would follow: transverse grains, carbides, and longitudinally oriented grains, with transverse grain boundaries being the most detrimental and longitudinal grains being the most beneficial, which is well documented in the literature $[4,14,17]$

\section{Carbide Fracture Behavior}

The results of the fractography clearly show that carbides are affecting the fracture behavior of the material. To identify the mechanism by which this fracture occurs, the scanning electron microscope montage of each set of mating fracture surfaces were examined side-by-side. The benefit of this is that one can clearly see if the carbides are debonding from the matrix or are themselves cracking into two halves that can be observed on both surfaces. For the material in this study, it is clear that the latter case occurs. Figure 11 shows a typical set of mating fracture surfaces. The carbides of interest can be seen on both sides of the fracture indicating that they have indeed cracked rather than debonded. 

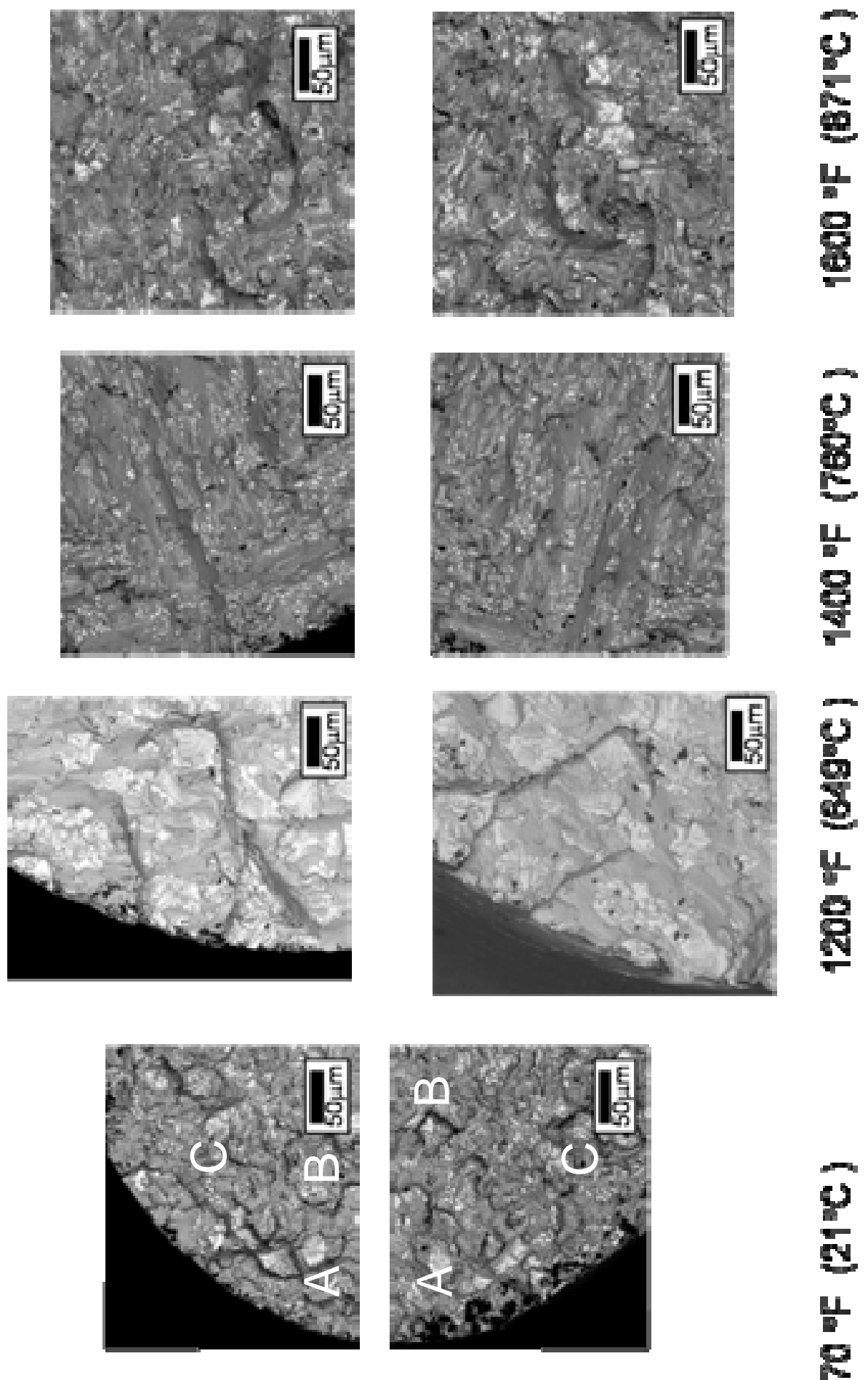

Figure 13: BSE images of mating surfaces of longitudinal tensile specimens $25,649,860$, and $881^{\circ} \mathrm{C}$. Points $\mathrm{A}, \mathrm{B}$, and $\mathrm{C}$ represents areas in which cracked carbides can be observed on both surfaces. This suggests that the carbides are not delaminating during failure. 


\section{Conclusions}

Statistical scatter in tensile tests performed by Ibanez [1], as well as a qualitative observation of an abundance of carbides on the fracture surfaces of each specimen has led to the need to quantify the anisotropy of GTD-111 DS as well as the role of carbides on fracture for both orientations of tensile tests. The major points of this paper are:

(1) Due to the partially oriented structure of GTD-111 DS, samples sectioned in the transverse direction with respect to the growth axis will have approximately four times as many grain boundaries per unit length than longitudinally oriented samples. These grain boundaries are perpendicular to the loading axis and based on creep elongation data are weaker than longitudinally oriented grain boundaries.

(2) Profile analysis has shown that for tensile tests conducted at temperatures of $25,649,860$, and $881^{\circ} \mathrm{C}$ the true area fraction of carbides on the fracture surface is significantly higher than $2 \%$, the volume fraction found in the bulk material, for longitudinally oriented samples.

(3) For transversely oriented samples, where profilemetry could not be employed, laser confocal microscopy was employed to determine the surface roughness number of the samples and calculate the true area fraction, and determine the number density of carbides on the fracture surface. Again, these values are much greater than those found in the bulk material. This indicates that the carbides clearly influence the fracture of the material in both orientations. However it is important to note that there was a greater amount of carbides found in the longitudinal case, which is attributed to the fact that transversely oriented grains are weak and cause failure, whereas failure of longitudinally oriented boundaries, which are stronger, must be assisted by the presence of the carbides.

(4) Examination of mating surfaces show that carbides are cracking rather than debonding from the matrix, which may hold true for crack initiation under other loading conditions such as fatigue.

\section{References}

[1] A. R. Ibanez, PhD Dissertation, Georgia Institute of Technology, May 2003.

[2] E. Huron, Master Dissertation, Georgia Institute of Technology, March 1986.

[3] W. Milligan, PhD Dissertation, Georgia Institute of Technology, 1988.

[4] K.L Gasko G.M. Janowski and B.J. Pletka. Materials Science and Engineering A, 104:1-8, 1988.

[5] R. Leverant and M. Gell. The elevated temperature fatigue of a nickel-based superalloy, mar-m200 in conventionally-cast and directionally solidified forms. Transaction of the Metallurgical Society AIME, 245:1168-1183, June 1969.

[6] W.F. Smith, Structure and Properties of Engineering Alloys 2nd Ed, McGraw-Hill (1993).
7] A.R. Ibanez, A Saxena, JD Kang, Strength, Fracture and Complexity, IOS Press, Volume 4, Number 2 p. 85 - 81, (2006).

[8] Arun M. Gokhale,AMS Handbook,ASM International, Materials Park, OH, 9 p. 428-448, (2004)

[9] Arun M. Gokhale,AMS Handbook,ASM International, Materials Park, OH, 11 p. 538-556, (2002).

[10] K.E. Kurtis N.H. El-Ashkar C.L. Collins, N.N.Naik, Cement and Concrete Composites, 25 p. 695-801. (2003).

[11] D.A. Woodford and A. Stiles, Journal of Materials Engineering and Performance, 6, p. 521-533,(1998).

[12] J.A. Daleo, K.A. Ellison, and D.A. Woodford, Journal of Engineering of Gas Turbines and Power, 6, p. 129-138,(1999).

[13] M. Trexler, B. Church, T. Sanders, Jr, Scripta Materialia, Volume 55, Issue 6, Pages 561-564, (2006).

[14] C. T. Sims, N. S. Stoloff, W. C. Hagel, Superalloys II, John Wiley and Sons,(1988).

[15] M.J. Donachie Jr. and O.H. Kreige, Journal Of Materials, 8(3) p. 269288, September, (1982).

[16] J. Goldstein D. Newbury P. Echlin D. Joy A. Romig C Lyman C. Fior and E. Lifshin, Scanning Electron Microscopy and X-ray Microanalysis,Plenum Press,(1992).

[17] M. Durand-Durand-Charre, The Microstructure of Superalloys, Gordon and Breach Science Publishers, (1998).

\section{Acknowledgments}

The authors would like to thank Dr. Alejandro Ibanez for the use of his tensile samples and data following the completion of his doctoral work and Dr. Kimberly Kurtis for use of the laser confocal microscope. The work discussed in this paper is the result of partial support from General Electric Energy and the Department of Energy 